\title{
Deformed dispersion relations and the degree of coherence function
}

\author{
Abel Camachd* and Alfredo Macías \\ Departamento de Física, Universidad Autónoma Metropolitana-Iztapalapa \\ Apartado Postal 55-534, C.P. 09340, México, D.F., México.
}

(Dated: September 15, 2017)

\begin{abstract}
The analysis of the modifications that the presence of a deformed dispersion relation entails in the roots of the so-called degree of coherence function, for a beam embodying two different frequencies and moving in a Michelson interferometer, is carried out. The conditions to be satisfied, in order to detect this kind of quantum gravity effect, are also obtained.
\end{abstract}

PACS: 04.80.Cc

\section{INTRODUCTION}

Amid the gamut of approaches, whose goal is a quantum theory of gravity, some clearly protrude, namely, they entail the modification of the dispersion relation [1]. These ideas appear in several models, for instance, quantumgravity approaches based upon non-commutative geometry [2, 3], or loop-quantum gravity models [4, 5], etc. In them Lorentz symmetry becomes only an approximation for quantum space [6, 7, 8], and do entail modifications in some fundamental physical concepts, as the uncertainty principle $[9]$, for instance.

The quest for quantum-gravity effects is not restricted to the case of dispersion relations. Indeed, Dirac equation can also be employed [10] in this context, and hence we may look for the consequences of this sort of models in the motion equation of $1 / 2$ spin particles. For instance, the spreading of a wave packet is modified, and in principle, it is possible to detect effects induced by quantum-gravity theory by monitoring this parameter, or, as already shown [10], Larmor precession presents a novel dependence, and in consequence the angular velocity of the expectation values of the components of the spin allows us to test our modified Dirac equation.

In the present work the consequences, upon the roots of the so-called degree of coherence function 11], of a deformed dispersion relation are analyzed. This will be done for the case of a beam comprising two different frequencies. At this point it is noteworthy to comment the existence, already, of a work 12] containing a qualitative analysis of the modifications emerging in the interference pattern of a Michelson device. In the aforementioned work the modifications in the phase shifts have been studied, nevertheless the changes in the roots of the degree of coherence function has not yet been addressed. The conditions to be satisfied, in order to detect this kind of quantum gravity effect, will be also deduced.

Finally, some words will be said concerning the possible future work in the realm of deformed dispersion relations and its detection resorting to higher-order coherence effects (such as the so-called Hanbury-Brown-Twiss effect [1]]), or to non-monocromatic light sources, a case that, by the way, up to now strangely has not been seriously considered, and the one could shed some light upon the present issue.

\section{DEGREE OF COHERENCE FUNCTION AND DEFORMED DISPERSION RELATIONS}

As already mentioned above several quantum-gravity models predict a modified dispersion relation [1, 2, 3, 4, 5], the one can be characterized, phenomenologically, through corrections hinging upon Planck's length, $l_{p}$,

$$
E^{2}=p^{2}\left[1-\alpha\left(E l_{p}\right)^{n}\right]
$$

\footnotetext{
*Electronic address: acq@xanum.uam.mx

† Electronic address: amac@xanum.uam.mx
} 
Here $\alpha$ is a coefficient, usually of order 1 , and whose precise value depends upon the considered quantum-gravity model, while $n$, the lowest power in Planck's length leading to a non-vanishing contribution, is also model dependent. Casting (1) in ordinary units we have

$$
E^{2}=p^{2} c^{2}\left[1-\alpha\left(E \sqrt{G /\left(c^{5} \hbar\right)}\right)^{n}\right]
$$

The expression

$$
p=\hbar k
$$

leads us to

$$
k=\frac{E /(c \hbar)}{\left[1-\alpha\left(E \sqrt{G /\left(c^{5} \hbar\right)}\right)^{n}\right]^{1 / 2}} .
$$

Since we expect very tiny corrections, then the following expansion is justified

$$
k=\frac{E}{c \hbar}\left[1+\frac{\alpha}{2}\left(E \sqrt{G /\left(c^{5} \hbar\right)}\right)^{n}+\frac{3}{8} \alpha^{2}\left(E \sqrt{G /\left(c^{5} \hbar\right)}\right)^{2 n}+\ldots\right] .
$$

Let us now consider two beams with energies $E_{1}$ and $E_{2}$, respectively, such that $E_{2}=E_{1}+\Delta E$, and in addition, it will be assumed that they interfere in a Michelson device [11. As is already known each frequency produces an interference pattern, and at this point it will be supposed that the corresponding beat frequency is to high to be detected [1], i.e., the output intensity is obtained adding the intensities associated with each frequency contained in the input. Under these conditions the measured intensity reads

$$
I=I_{1}\left[1+\cos \left(\omega_{1} \tau_{1}\right)\right]+I_{2}\left[1+\cos \left(\omega_{2} \tau_{2}\right)\right]
$$

In this last expression $I_{1}$ and $I_{2}$ denote the intensities of the two beams, $\omega_{1}, \omega_{2}$ the corresponding frequencies, and

$$
\tau_{1}=2 d / c_{1}, \quad \tau_{2}=2 d / c_{2}
$$

Here $d$ is the difference in length in the two interferometer arms, and $c_{1}, c_{2}$, the corresponding velocities, here the velocity has a non-trivial energy dependence 1], i.e., $c_{1} \neq c_{2}$.

From now on we will assume that $I_{1}=I_{2}$, such that $I_{0}=I_{1}+I_{2}$, therefore the detected intensity can be cast in the following form

$$
I=I_{0}[1+\gamma(d)]
$$

In this last equation the so-called degree of coherence function $\gamma(d)$ has been introduced [11], the one for our situation reads ( $k_{1}$ and $k_{2}$ are the corresponding wave numbers)

$$
\gamma(d)=\cos \left(\left[k_{1}+k_{2}\right] d / 2\right) \cos \left(\left[k_{1}-k_{2}\right] d / 2\right)
$$

A fleeting glimpse at (4) clearly shows that (9) does depend upon $\alpha$ and $n$, and in consequence the roots of the degree of coherence function will be modified by the presence of a deformed dispersion relation.

The expression providing us the roots of the degree of coherence function is

$$
\left(k_{1}-k_{2}\right) d / 2=\pi / 2
$$

Resorting to our previous expressions we may rewrite (10) as 


$$
\begin{array}{r}
d=c \hbar \pi\left\{\Delta E+\frac{\alpha}{2} E_{1}\left(E_{1} \sqrt{G /\left(c^{5} \hbar\right)}\right)^{n}\right. \\
\left.\times\left[(n+1) \frac{\Delta E}{E_{1}}+\frac{n(n+1)}{2}\left(\frac{\Delta E}{E_{1}}\right)^{2}+\ldots\right]\right\}^{-1} .
\end{array}
$$

Let us now define $\beta=\Delta E / E_{1}$, a real number smaller than 1 . In the present proposal we will consider two possible values for $n$, namely:

\section{A. Case $n=1$}

For this situation we have that the roots of the degree of coherence function become, approximately

$$
d=\frac{c \hbar \pi}{E_{1}}\left\{\beta-\frac{\alpha}{2}\left(E_{1} \sqrt{G /\left(c^{5} \hbar\right)}\right)[2+\beta]\right\} .
$$

For the sake of clarity let us assume that $\alpha \sim 1$, a restriction that is not devoid of physical content [1]. The possibility of detecting this deformed dispersion relation will hinge upon the fulfillment of the condition

$$
|D-d|>\Delta d .
$$

In this last equation $D$ denotes the usual value in the difference of the interferometer arms at which the degree of coherence function vanishes (that is when $\alpha=0$ ), whereas $\Delta d$ is the corresponding experimental resolution. This can be cast in the following form

$$
\frac{\Delta E}{E_{1}}>\frac{2 \Delta d}{\pi l_{p}}-1
$$

Recalling that from square one it was assumed that our device cannot detect the beat frequencies, i.e., if $T$ denotes the time resolution of the measuring device, then

$$
\left|\omega_{2}-\omega_{1}\right| T / 2>>1 \text {. }
$$

This last condition may be rewritten as

$$
T \Delta E>\hbar \text {. }
$$

In other words, (14) and (16) are the two conditions to be fulfilled if the case $n=1$ and $\alpha \sim 1$ is to be detected.

\section{B. Case $n=2$}

Under these conditions ( $n=2$ and $\alpha \sim 1)$ the roots of the degree of coherence function read, approximately

$$
d=\frac{c \hbar \pi}{E_{1}}\left\{\beta-\frac{\alpha}{2}\left(E_{1} \sqrt{G /\left(c^{5} \hbar\right)}\right)^{2}\left[3+3 \beta+\beta^{2}\right]\right\} .
$$


The expression tantamount to (14) is

$$
\left\{3+3\left(\frac{\Delta E}{E_{1}}\right)+\left(\frac{\Delta E}{E_{1}}\right)^{2}\right\} E_{1}>2 \frac{c \hbar \Delta d}{\pi l_{p}^{2}} .
$$

The impossibility of detecting beat frequencies translates, once again, as

$$
T \Delta E>\hbar
$$

\section{CONCLUSIONS}

In the present work the possibility of detecting two different deformed dispersion relations, resorting to the analysis of the roots of the degree of coherence function, has been carried out.

The impossibility of detecting beat frequencies renders only one condition, see expressions (16) and (19). The experimental difficulty appears in connection with (14) and (18), which are the restrictions to be satisfied in order to

detect this kind of effects. Forsooth, since we have, from square one, imposed the condition $\frac{\Delta E}{E_{1}}<1$, then (14) entails a very stringent restriction, namely a experimental resolution very close to Planck's length, i.e., $\Delta d \sim l_{p}$.

The case $n=2$ becomes even worse, as usual [1]. A rough estimate of the required energy, for the case in which $\Delta d \sim 10^{-4} \mathrm{~cm}$, renders energies higher than the so-called GZK limit for cosmic rays 13. A fleeting glimpse at (18) clearly shows us that in this case the problem stems from the presence of the factor $l_{p}^{2}$.

The conclusions that have been drawn from the previous analysis have a not very optimistic atmosphere, and in consequence we may wonder if interferometry could be useful in the present context. In order to address this issue let us recall that in the extant literature the considered experimental proposals do reduce to the case of first order coherence experiments [12] (Michelson interferometer falls within this category 11]), or they consider only a finite number of monocromatic sources 12 . At this point it is noteworthy to comment that optical experiments offer a much richer realm of possibilities. Forsooth, higher-order coherence effects, for instance, the so called Hanbury-BrownTwiss effect [1] ]), could be also considered and explored within the present context, or the effects of a deformed dispersion relation upon a light source with a continuous frequency distribution could be studied. The results of the analysis of the aforementioned proposals will be published elsewhere.

\section{Acknowledgments}

We dedicate the present work to Michael Ryan on occasion of his $60^{\text {th }}$ birthday. This research was supported by CONACYT Grant 42191-F. A. C. would like to thank A.A. Cuevas-Sosa for useful discussions and literature hints.

[1] G. Amelino-Camelia, Int. J. Mod. Phys. D9, 1633 (2003).

[2] G. Amelino-Camelia and T. Piran, Phys. Rev. D64, 036005 (2001).

[3] G. Amelino-Camelia, Nature 418, 661 (2000).

[4] R.Gambini and J. Pullin, Phys. Rev. D59, 124021 (1999).

[5] L.Smolin, LANL hep-th/0303185

[6] G. Amelino-Camelia, Nature 398, 216 (1999).

[7] G. Amelino-Camelia, J. Ellis, N. E. Mavromatos, D. V. Nanopoulos, and S. Sarkar, Nature 393, 763 (1998).

[8] J. Alfaro, H. A. Morales-Tecotl, and L. F. Urrutia, Phys. Rev. Lett. 84, 2318 (2000).

[9] A. Camacho, Gen. Rel. Grav. 34, 1839 (2002).

[10] A. Camacho and A. Macias, Phys. Lett. B582, 229 (2004).

[11] L. Mandel and E. Wolf, Optical Coherence and Quantum Optics, Cambridge University Press, Cambridge (1995).

[12] G. Amelino-Camelia and C. Lämmerzahl, Class. Quantum Grav.21, 899-915 (2004).

[13] T. Piran, Gamma-Ray Bursts as Probes for Quantum Gravity, arXiv: astro-ph/0407462 